\title{
Geração de uma excitação sísmica através do espectro de Kanai-Tajimi
}

\author{
Daniela Dalla Chiesa ${ }^{1}$ \\ Letícia Fleck Fadel Miguel ${ }^{1}$
}

\begin{abstract}
Resumo: O presente artigo apresenta os resultados da implementação de uma rotina computacional, no programa MATLAB ${ }^{\circledR}$, para a geração de uma excitação sísmica e análise da resposta dinâmica de um edifício, com $n$ graus de liberdade, sujeito a esse tipo de carregamento. Define-se a excitação sísmica como um processo estocástico estacionário unidimensional, passando por um processo de ruído branco Gaussiano através do filtro de Kanai-Tajimi, cuja função densidade espectral de potência permite a geração da aceleração do solo no domínio da frequência. A aceleração do solo é, então, passada para o domínio do tempo gerando um acelerograma sísmico artificial. De posse do acelerograma resolve-se, com a aplicação do método de Newmark, a equação de movimento dinâmico estrutural e encontram-se os deslocamentos, velocidades e acelerações referentes a cada andar do edifício. Por fim, uma análise dos valores máximos, em módulo, dos deslocamentos, acelerações e dos deslocamentos relativos entre os andares é ilustrada. Os resultados encontrados estão condizentes com a literatura confirmando, assim, a eficiência da rotina implementada.
\end{abstract}

Palavras-chave: Excitação sísmica. Kanai-Tajimi. MATLAB ${ }^{\circledR}$.

\begin{abstract}
This paper presents the results of a computational routine implementation in the $M A T L A B^{\circledR}$ software for the generation of a seismic excitation and dynamic response analysis of a building, with $n$ degrees of freedom, subject to this loading type. The seismic excitation is defined as an one-dimensional stochastic process, that is simulated undergoing by a Gaussian white noise process through a Kanai-Tajimi filter, which the power spectral density function generates the ground acceleration in the frequency domain. The ground acceleration is passed to the time domain generating an artificial seismic accelerogram. With the artificial seismic accelerogram and the application of the Newmark method, the structural dynamic motion equation is solved and the displacements, velocities and accelerations in each building floor are found. Finally, an analysis of the maximum values, in modulus, of the displacements, accelerations and drift is illustrated. The results are in agreement with the literature, confirming the efficiency of the implemented routine.
\end{abstract}

Keywords: Kanai-Tajimi. MATLAB ${ }^{\circledR}$. Seismic excitation.

\section{Introdução}

Todos os dias têm-se registros de abalos sísmicos no mundo. A maior parte desses registros, no entanto, não é sentida pelos seres humanos devido a fatores como a magnitude ou a distância epicentral, entre outros. Entretanto, alguns terremotos ficaram marcados na história da humanidade devido ao grande número de vítimas e perdas materiais provocadas, em sua grande maioria, porque as construções dos locais atingidos não foram capazes de resistir ao tremor de terra induzido pelo abalo sísmico, como exemplos têm-se os terremotos ocorridos no Haiti, em 2010, cujo número de mortos foi de 316.000 pessoas, seguido pelos terremotos ocorridos na costa oeste da Sumatra do Norte, em 2004, e Sichuan Oriental na China, em 2008, cujo número de mortos foi de 227.898 e 87.587 pessoas, respectivamente. Um terremoto, seguido de um tsunami, que vitimou 20.896 pessoas e deixou muitas comunidades em ruínas acarretando, ao governo japonês, gastos de bilhões de dólares para recuperar as regiões atingidas foi o ocorrido próximo à costa leste de Honshu no Japão, em 2011, com magnitude de $9.0 M_{\mathrm{w}}$.

${ }^{1}$ Programa de Pós-Graduação em Engenharia Mecânica (PROMEC), UFRGS, Rua Sarmento Leite, 425 - Centro Histórico - Porto Alegre (RS) - Brasil

\{ddchiesa@gmail.com, letffmeufrgs.br\}

http://dx.doi.org/10.5335/rbca.v9i1.6660

Revista Brasileira de Computação Aplicada (ISSN 2176-6649), Passo Fundo, v. 9, n. 1, p. 31-41, abr. 2017 
Esse terremoto foi o segundo registrado de maior magnitude, entre os anos de 1990 a 2014, perdendo apenas para o ocorrido na costa oeste da Sumatra do Norte, em 2004, com a magnitude $9.1 M_{\mathrm{w}}$ [1].

Uma vez que a previsão de um terremoto é uma tarefa difícil e, até o momento, nenhum modelo probabilístico é capaz de determinar com precisão o horário ou a magnitude do evento, pesquisas no que se referem à elaboração de projetos estruturais que sejam resistentes a eventos sísmicos, os chamados projetos sismo-resistentes, têm se mostrado uma opção viável na redução de mortes e danos materiais.

Durante um terremoto, a base de uma estrutura é submetida à aceleração do solo promovida pelo sismo e esse movimento provoca na estrutura esforços e deformações. Consequentemente, para a elaboração de um projeto sismo-resistente é necessário construir um modelo probabilístico que descreva, de forma mais realista, as características da excitação sísmica no local em estudo. A NBR 15.421 [2], por exemplo, considera um modelo estático equivalente para a representação de um evento sísmico, entretanto, este modelo está distante de uma representação rigorosa e pode-se dizer que, mesmo para um país que situa-se fora de uma zona de atividade sísmica relevante, esse tipo de análise não é adequada, tendo em vista a natureza dinâmica do fenômeno.

Características de eventos sísmicos, como a aleatoriedade no tempo e no espaço, devem ser consideradas na elaboração de um projeto sismo-resistente, de modo que, esse tipo de análise tem por base a utilização de métodos estatísticos, provenientes da teoria da probabilidade.

No desenvolvimento probabilístico para vibração, tanto a excitação como a resposta estrutural são modeladas como processos estocásticos. Um processo estocástico pode ser especificado em termos de um número relativamente pequeno de parâmetros estatísticos e funções, como a função densidade espectral de potência [3], também denominada espectro de potência. Com tais características, vários métodos para a geração de abalos sísmicos foram desenvolvidos ao longo dos anos. Um dos métodos, que tem sido muito bem aceito pela comunidade científica e, em vista disso, adotado em vários trabalhos (por exemplo: [4], [5] e [6]) é o que utiliza a função densidade espectral de potência de Kanai-Tajimi [7] e [8] para a geração do sinal sísmico no domínio da frequência. Com o espectro de potência de Kanai-Tajimi, a aceleração do solo é simulada por meio de um processo estocástico estacionário de ruído branco Gaussiano através do filtro de Kanai-Tajimi.

Nesse contexto, o presente artigo apresenta os resultados de um estudo que teve por objetivo utilizar o programa MATLAB ${ }^{\circledR}$, como ferramenta para implementar uma rotina computacional, tanto para geração de uma excitação sísmica, utilizando o espectro de potência Kanai-Tajimi, quanto para análise da resposta dinâmica de um edifício sujeito a esse tipo de excitação. Na rotina elaborada, inicialmente, a aceleração do solo é gerada, no domínio da frequência, com a função densidade espectral de potência de Kanai-Tajimi, e, então, esse sinal é passado para o domínio do tempo, através da equação proposta por [9]. Posteriormente, aplica-se essa excitação na base de um edifício, com $n$ graus de liberdade (GDL), e analisa-se a sua resposta dinâmica, por meio do método de Newmark. A validação da rotina implementada é feita mediante a comparação dos resultados obtidos neste trabalho com os obtidos por [5]. A comparação de ambos os resultados permitem concluir que a rotina computacional implementada está condizente e, em virtude disso, poderá servir como alicerce para a elaboração, pelos autores, de modelos probabilísticos mais complexos, considerando processos estocásticos não-estacionários e estruturas tridimensionais.

\section{Processo estocástico}

Para um determinado fenômeno considere que será definido um experimento e que esse experimento pode ser repetido $N$ vezes em condições idênticas. Toda a vez que esse experimento for realizado uma função do tempo ${ }^{2}$, denominada função-amostra, será obtida como resultado, Figura 1. Cada função-amostra obtida, em geral, será diferente toda vez que o experimento for efetuado e, portanto, ter-se-á um conjunto infinito (teoricamente) de funções-amostra. A este conjunto infinito refere-se como um processo estocástico ou aleatório [10], [11] e [3].

Um fenômeno, representado por um processo estocástico, possui propriedades que podem, hipoteticamente, serem descritas em qualquer instante $t_{s}(s=1,2, \ldots)$, pela avaliação das médias das funções-amostra obtidas. Como exemplo, considere o processo estocástico $p(t)$, Figura 1. O valor médio de $p(t)$, em qualquer instante,

\footnotetext{
${ }^{2} \mathrm{Na}$ maioria das vezes a função obtida está relacionada com o tempo [10]. Em alguns problemas, entretanto, é conveniente considerar funções do espaço e tempo [3].
}

Revista Brasileira de Computação Aplicada (ISSN 2176-6649), Passo Fundo, v. 9, n. 1, p. 31-41, abr. 2017 
Figura 1: Funções-amostra do processo estocástico $p(t)$.
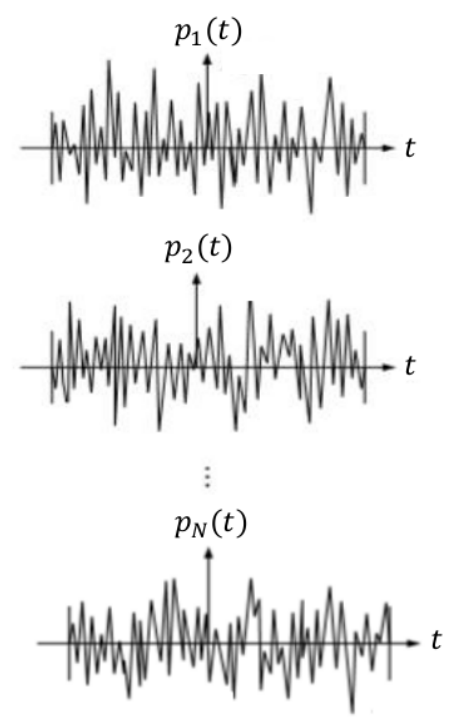

pode ser calculado somando os valores de cada função-amostra, do conjunto, no instante $t_{s} \mathrm{e}$, posteriormente, dividindo o resultado obtido pelo número de funções-amostra $p_{m}$, conforme descrito em (1) [11]:

$$
\mu_{p}\left(t_{s}\right)=\lim _{N \rightarrow \infty} \frac{1}{N} \sum_{m=1}^{N} p_{m}\left(t_{s}\right),
$$

onde $\mu_{p}\left(t_{s}\right)$ representa o valor médio de $p(t)$ no instante $t_{s}$.

De maneira similar, pode-se calcular a correlação, denominada função autocorrelação, de $p(t)$, através da média do produto em dois instantes quaisquer, $t_{s}$ e $t_{s}+\tau$, onde $\tau$ representa um incremento de tempo, de acordo $\operatorname{com}(2)[11]$ :

$$
R_{p}\left(t_{s}, t_{s}+\tau\right)=\lim _{N \rightarrow \infty} \frac{1}{N} \sum_{m=1}^{N} p_{m}\left(t_{s}\right) p_{m}\left(t_{s}+\tau\right),
$$

em que $R_{p}\left(t_{s}, t_{s}+\tau\right)$ é a função de autocorrelação em dois instantes distintos.

Para o caso em que $\mu_{p}\left(t_{s}\right)$ e $R_{p}\left(t_{s}, t_{s}+\tau\right)$ não variam com o tempo, ou seja, a média é constante e a função autocorrelação depende somente de $\tau\left(R_{p}(\tau)\right)$, o processo estocástico é denominado processo estocástico estacionário no sentido amplo [10] e [11].

\subsection{Densidade espectral de potência}

A densidade espectral de potência de um sinal, quando integrada ao longo de uma faixa de frequências fornece o valor da potência de sinal existente na faixa de frequências consideradas [10].

No caso de processos estocásticos encontram-se duas dificuldades quando trata-se de encontrar a composição de frequências do mesmo: A primeira se refere ao fato de que não é possível a sua representação pela série discreta de Fourier uma vez que os históricos de tempo das funções-amostra são aperiódicos. A segunda se deve ao fato que no processo estocástico estacionário a condição, dada por (3), não é satisfeita e, assim, a análise clássica da teoria de Fourier não pode ser aplicada [12].

$$
\int_{-\infty}^{\infty}|p(t)| \mathrm{d} t<\infty
$$

Revista Brasileira de Computação Aplicada (ISSN 2176-6649), Passo Fundo, v. 9, n. 1, p. 31-41, abr. 2017 
A segunda dificuldade, entretanto, pode ser superada analisando, não as funções-amostra do processo, mas a função de autocorrelação. A função de autocorrelação fornece informações sobre as frequências do processo estocástico indiretamente. A função $R_{p}(\tau)$ é um máximo para os valores de $\tau$ para quais $p\left(t_{s}\right)$ e $p\left(t_{s}+\tau\right)$ estão em fase e um mínimo para os valores de $\tau$ para quais eles estão em antifase, assim, as frequências presentes no gráfico de $R_{p}(\tau)$ versus $\tau$ refletem o conteúdo de frequências das funções-amostra do processo $p(t)$. Dessa forma, se o valor inicial de $p(t)$ é normalizado, de modo que o valor médio do processo seja zero, então, assumindo que $p(t)$ não tenha componentes periódicos [12]:

$$
R_{p}(\tau \rightarrow \infty)=0
$$

e assim a condição:

$$
\int_{-\infty}^{\infty}\left|R_{p}(\tau)\right| \mathrm{d} t<\infty
$$

é satisfeita e, no caso de processos estocásticos estacionários no sentido amplo, a densidade espectral de potência $S_{p}(\omega)$ é dada pela transformada de Fourier da sua função autocorrelação ${ }^{3}$ :

$$
S_{p}(\omega)=\frac{1}{2 \pi} \int_{-\infty}^{\infty} R_{p}(\tau) e^{-i \omega \tau} \mathrm{d} \tau,
$$

onde $\omega$ é a frequência angular e $i=\sqrt{-1}$. A sua inversa é expressa por:

$$
R_{p}(\tau)=\int_{-\infty}^{\infty} S_{p}(\omega) e^{i \omega \tau} \mathrm{d} \omega
$$

\section{Equação de um sistema dinâmico sujeito a uma excitação sísmica}

A equação de movimento de um sistema, com $n$ GDL, sujeito a uma excitação sísmica em sua base pode ser expressa por [13] e [4]:

$$
\mathbf{M} \ddot{\mathbf{z}}(t)+\mathbf{C} \dot{\mathbf{z}}(t)+\mathbf{K z}(t)=\mathbf{F}(t),
$$

em que $\mathbf{M}, \mathbf{C}$ e $\mathbf{K}$ são as matrizes, de ordem $n$, de massa, amortecimento e rigidez, respectivamente, $\ddot{\mathbf{z}}(t), \dot{\mathbf{z}}(t)$ e $\mathbf{z}(t)$, são, nesta ordem, os vetores de aceleração, velocidade e deslocamento relativos do sistema, isto é, do movimento do sistema em relação a sua base, e $\mathbf{F}(t)$ é o vetor de forças externas expresso por:

$$
\mathbf{F}(t)=-\mathbf{M B} \ddot{\mathbf{y}}(t),
$$

onde $\mathbf{B}$ é a matriz $n \mathrm{x} d$ que contém os cossenos diretores dos ângulos formados entre o movimento da base e as direções dos GDL, $d$ é o número de movimentos considerados e $\ddot{\mathbf{y}}(t)$ representa o vetor de aceleração do solo, ou seja, a representação da excitação sísmica.

O sinal negativo, em geral, tem pouca relevância na análise de resposta de um sistema sujeito a uma excitação sísmica e, portanto, pode ser removido [13].

\section{Geração da excitação sísmica}

A aceleração do solo, no domínio da frequência, é definida por um processo estocástico estacionário unidimensional passando por um processo de ruído branco Gaussiano através do filtro de Kanai-Tajimi [7] e [8], com função densidade espectral de potência $S(\omega)$ expressa por:

$$
S(\omega)=S_{0}\left[\frac{\omega_{s}^{4}+4 \omega_{s}^{2} \xi_{s}^{2} \omega^{2}}{\left(\omega^{2}-\omega_{s}^{2}\right)^{2}+4 \omega_{s}^{2} \xi_{s}^{2} \omega^{2}}\right] \text { e } S_{0}=\frac{0,03 \xi_{s}}{\pi \omega_{s}\left(4 \xi_{s}^{2}+1\right)},
$$

onde $S_{0}$ é a densidade espectral constante, $\omega_{s}$ e $\xi_{s}$ são, nesta ordem, a frequência e o amortecimento do solo, cujas características dependem da região em análise. A Figura 2 ilustra um espectro de potência gerado com o filtro de Kanai-Tajimi.

${ }^{3}$ Trata-se do Teorema de Wiener-Khintchine. 
Figura 2: Espectro de potência gerado com o fitro de Kanai-Tajimi para $\omega_{s}=37,3 \mathrm{rad} / \mathrm{s}$ e $\xi_{s}=0,3$.

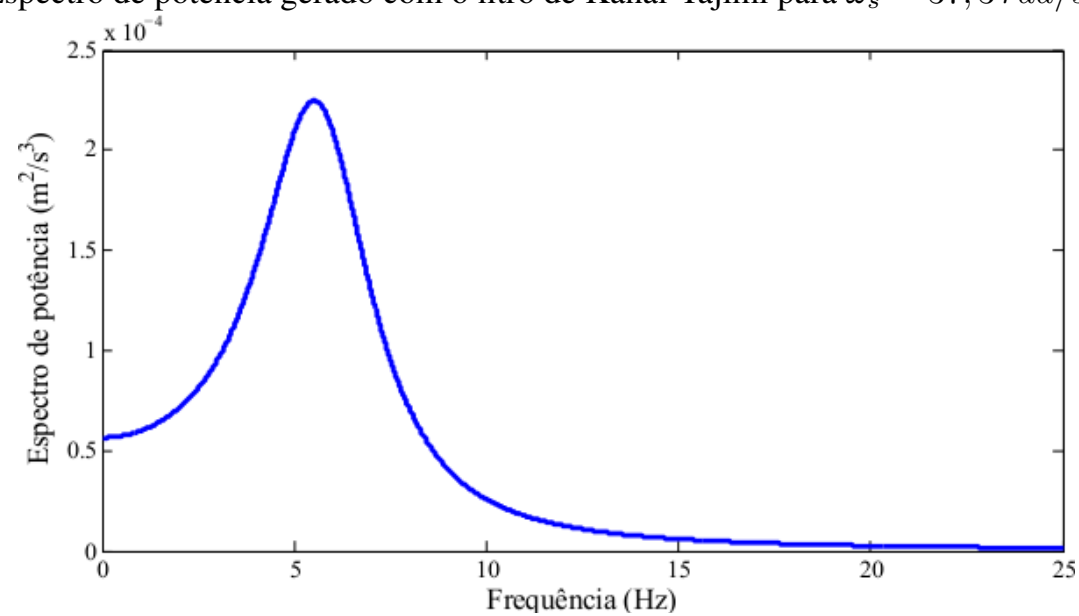

A solução de (10) permite a geração de um sinal para a aceleração do solo no domínio da frequência, portanto, com o objetivo de passar esse mesmo sinal para o domínio do tempo, ou obter-se um acelerograma sísmico artificial, é utilizado o método proposto por [9], cuja equação é dada por:

$$
\ddot{\mathbf{y}}(t)=\sqrt{2} \sum_{j=1}^{N_{\omega}} \sqrt{S\left(\omega_{j}\right) \Delta \omega} \cos \left(w_{j} t+\phi_{j}\right),
$$

onde $N_{\omega}$ é o número de intervalos da banda de frequências, $\Delta \omega$ é o incremento de frequências e $\phi_{j}$ é o ângulo de fase, cujos valores são escolhidos, de forma aleatória, com distribuição uniforme entre 0 e $2 \pi$.

Após a obtenção do sinal, no domínio do tempo, ainda, é necessário normalizar esses valores, de forma que o valor de pico de aceleração do solo ${ }^{4}$ esteja de acordo com as características do solo da região em estudo.

\section{Método de Newmark}

A abordagem numérica torna-se necessária quando a equação diferencial de movimento de um sistema vibratório não pode ser integrada de forma analítica, sendo assim, (8) pode ser resolvida utilizando algum método de integração numérica.

Os métodos de integração numérica possuem duas características relevantes. A primeira consiste em satisfazer a equação em questão em somente intervalos de tempos discretos separados por $\Delta t$, ao invés de satisfazê-la em todos os tempos. A segunda admite algum tipo de variação para o deslocamento, a velocidade e a aceleração, dentro de cada intervalo de tempo $\Delta t$ [14].

Entre os diferentes métodos de integração numérica tem-se o método de Newmark. O método de Newmark é um método implícito de integração que se baseia no fato de que a aceleração varia linearmente entre dois instantes de tempo.

O método parte do pressuposto que os valores de deslocamento e velocidade em $t=0$ são conhecidos e, então, calcula-se a aceleração inicial através da equação:

$$
\ddot{\mathbf{z}}\left(t_{0}\right)=\mathbf{M}^{-1}\left[\mathbf{F}\left(t_{0}\right)-\mathbf{C} \dot{\mathbf{z}}\left(t_{0}\right)-\mathbf{K} \mathbf{z}\left(t_{0}\right)\right] .
$$

De posse dessas informações determina-se a solução da equação diferencial de movimento no intervalo de $t=0$ a $t=T$, onde $T$ indica a duração de tempo. As expressões para os cálculos de deslocamentos, velocidades

\footnotetext{
${ }^{4}$ Em inglês Peak Ground Acceleration (PGA).
} 
e acelerações, referentes à (8), são dadas, respectivamente, por:

$$
\begin{gathered}
\mathbf{z}\left(t_{j+1}\right)=\mathbf{D}\left\{\mathbf{F}\left(t_{j+1}\right)+\mathbf{M}\left[a_{0} \mathbf{z}\left(t_{j}\right)+a_{1} \dot{\mathbf{z}}\left(t_{j}\right)+a_{2} \ddot{\mathbf{z}}\left(t_{j}\right)\right]+\mathbf{C}\left[a_{3} \mathbf{z}\left(t_{j}\right)+a_{4} \dot{\mathbf{z}}\left(t_{j}\right)+a_{5} \ddot{\mathbf{z}}\left(t_{j}\right)\right]\right\} \mathrm{e} \\
\mathbf{D}=\left(a_{0} \mathbf{M}+a_{3} \mathbf{C}+\mathbf{K}\right)^{-1}, \\
\dot{\mathbf{z}}\left(t_{j+1}\right)=a_{3}\left[\mathbf{z}\left(t_{j+1}\right)-\mathbf{z}\left(t_{j}\right)\right]-a_{4} \dot{\mathbf{z}}\left(t_{j}\right)-a_{5} \ddot{\mathbf{z}}\left(t_{j}\right), \\
\ddot{\mathbf{z}}\left(t_{j+1}\right)=a_{0}\left[\mathbf{z}\left(t_{j+1}\right)-\mathbf{z}\left(t_{j}\right)\right]-a_{1} \dot{\mathbf{z}}\left(t_{j}\right)-a_{2} \ddot{\mathbf{z}}\left(t_{j}\right),
\end{gathered}
$$

onde:

$$
a_{0}=\frac{1}{\alpha \Delta t^{2}}, \quad a_{1}=\frac{1}{\alpha \Delta t}, \quad a_{2}=\frac{1}{2 \alpha}-1, \quad a_{3}=\frac{\beta}{\alpha \Delta t}, \quad a_{4}=\frac{\beta}{\alpha}-1, \quad a_{5}=\frac{\Delta t}{2}\left(\frac{\beta}{\alpha}-2\right) \text {, }
$$

em que $\alpha$ e $\beta$ são parâmetros que indicam quanto a aceleração ao final do intervalo entra nas equações de velocidade e deslocamento ao final do intervalo $\Delta t$ [14].

\section{Validação da rotina computacional}

A rotina computacional implementada foi desenvolvida de forma a possibilitar o cálculo de deslocamentos, velocidades e acelerações unidimensionais, em intervalos de tempos discretos separados por $\Delta t$, de um edifício, com $n$ GDL, sujeito a uma excitação sísmica na sua base, conforme ilustrado na Figura 3.

Figura 3: Edifício com $n$ GDL, em que $\mathbf{w}(t)=\mathbf{z}(t)+B \mathbf{y}(t)$. Adaptado de Mohebbi et al. [5], 2013, p. 607.

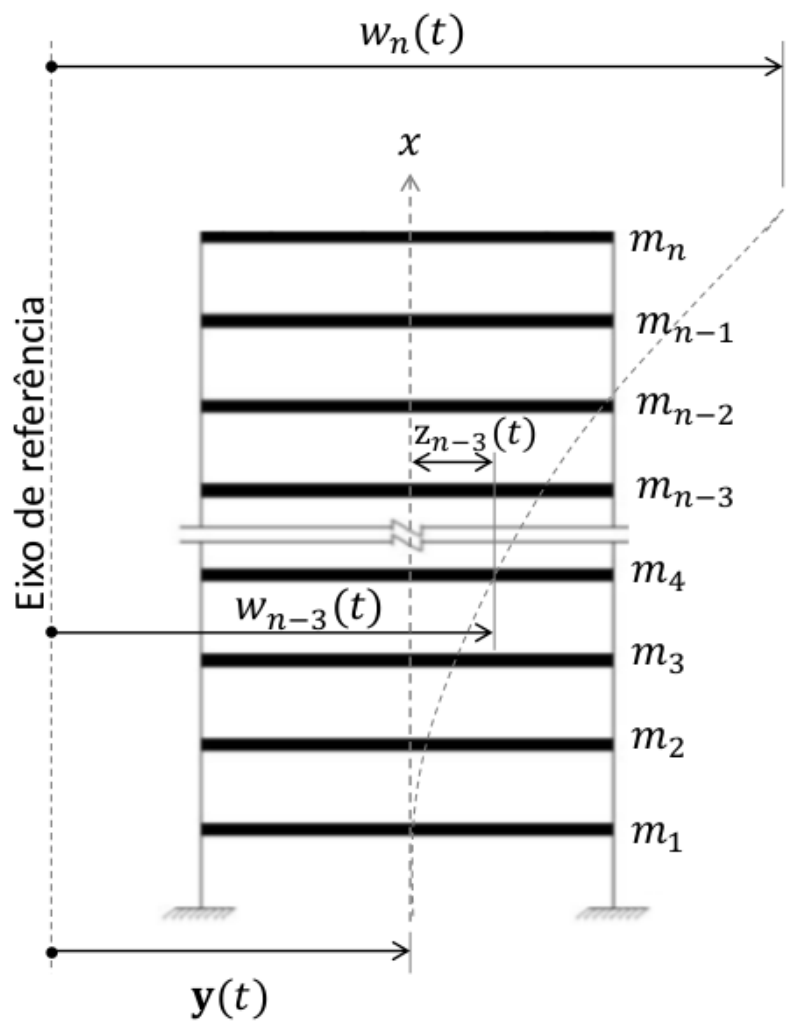

Quatro simulações numéricas foram realizadas, com os mesmos parâmetros adotados por [5], com o intuito de validar a rotina computacional. Para a geração do sinal sísmico, no domínio do tempo, os seguintes dados foram utilizados: frequência mínima $f_{\min }=0 \mathrm{~Hz}$, frequência máxima $f_{\max }=25 \mathrm{~Hz}$, incremento de frequência 
$\Delta f=0,01 \mathrm{~Hz}$, tempo inicial $t_{0}=0 \mathrm{~s}$, tempo final $T=50 \mathrm{~s}$, incremento de tempo $\Delta t=0,01 \mathrm{~s}$, amortecimento do solo $\xi_{s}=0,3$ e frequência do solo $\omega_{s}=37,3 \mathrm{rad} / \mathrm{s}$.

Na Figura 4 é exposto o histórico de aceleração do solo obtido por [5] e a na Figura 5 os históricos de acelerações do solo obtidos pelos autores. Os resultados apresentados estão normalizados com um valor de pico de aceleração do solo de $0,475 \mathrm{~g}$, onde $g$ indica a aceleração gravitacional $\left(g \approx 9,81 \mathrm{~m} / \mathrm{s}^{2}\right)$. A Figura 6 mostra os espectros de potências gerados nas simulações $1,2,3$ e 4 .

Figura 4: Sinal sísmico obtido por [5], com pico de aceleração do solo de 0,475g. Fonte: Mohebbi et al. [5], 2013, p. 611.

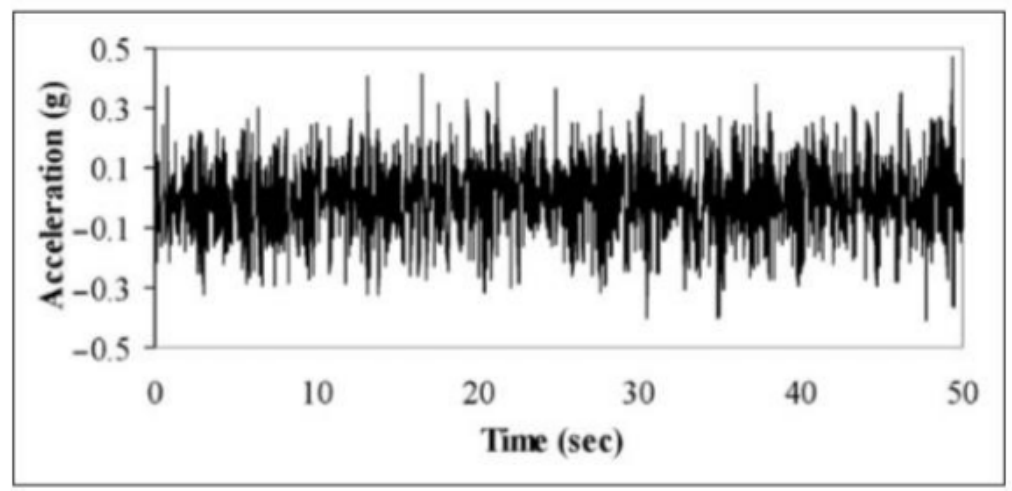

Figura 5: Sinais sísmicos obtidos através das simulações 1, 2, 3 e 4, com pico de aceleração do solo de $0,475 g$.

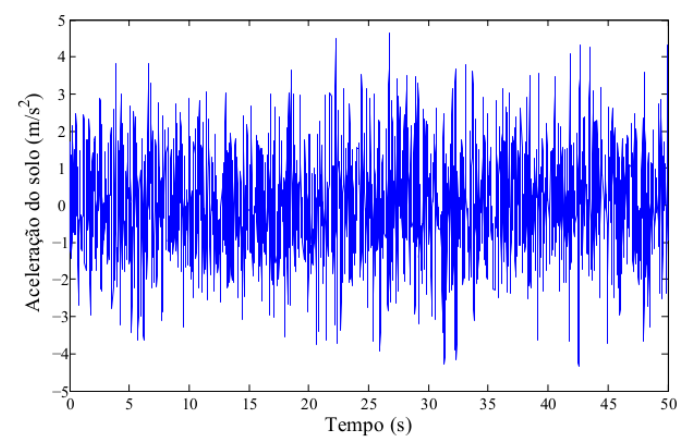

(a) Sinal sísmico obtido na simulação 1 .

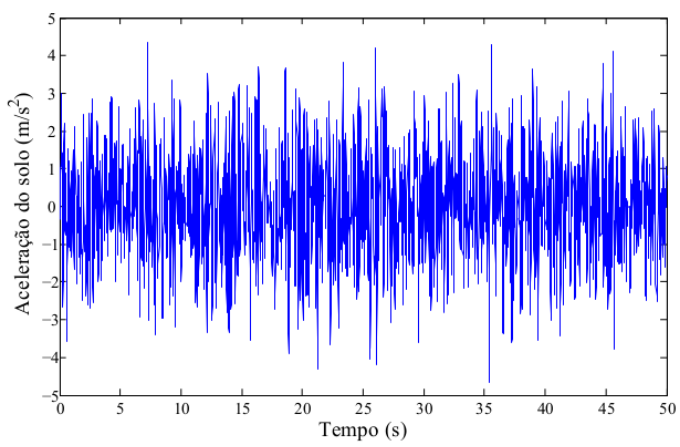

(c) Sinal sísmico obtido na simulação 3 .

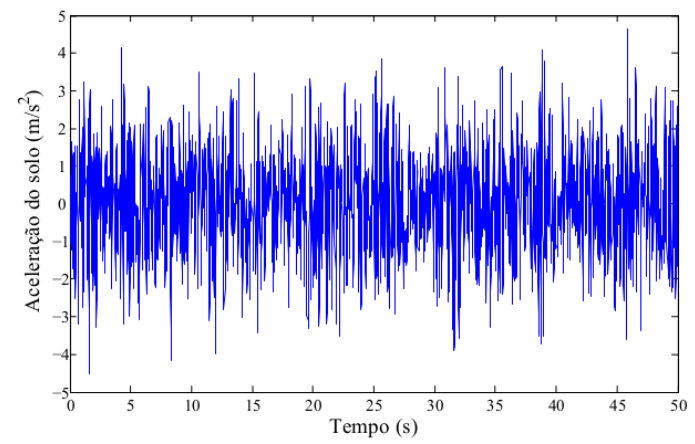

(b) Sinal sísmico obtido na simulação 2 .

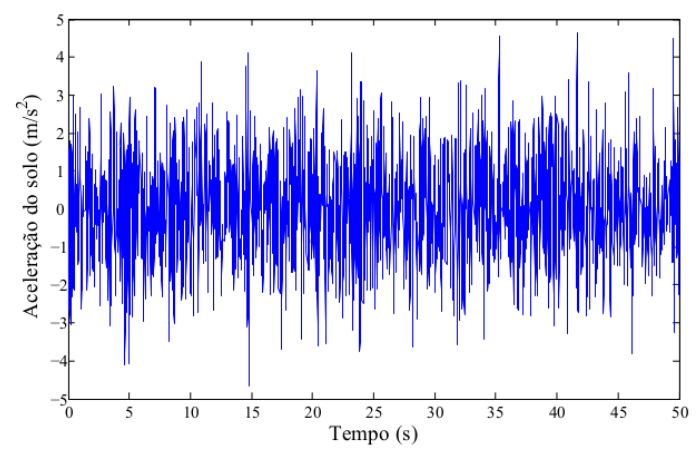

(d) Sinal sísmico obtido na simulação 4 . 
Figura 6: Espectros de potência obtidos através das simulações 1, 2, 3 e 4.

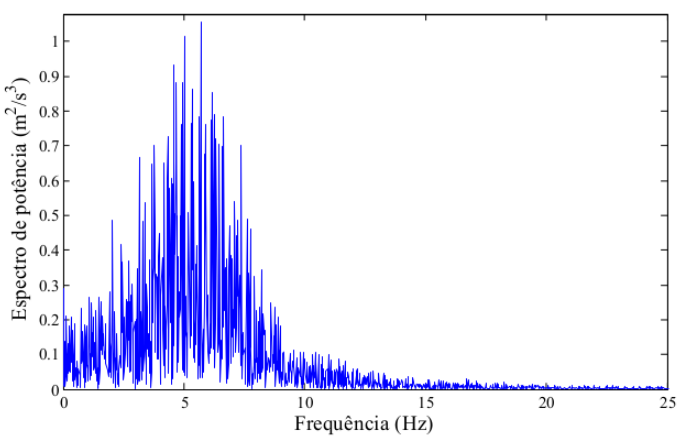

(a) Espectro de potência obtido na simulação 1.

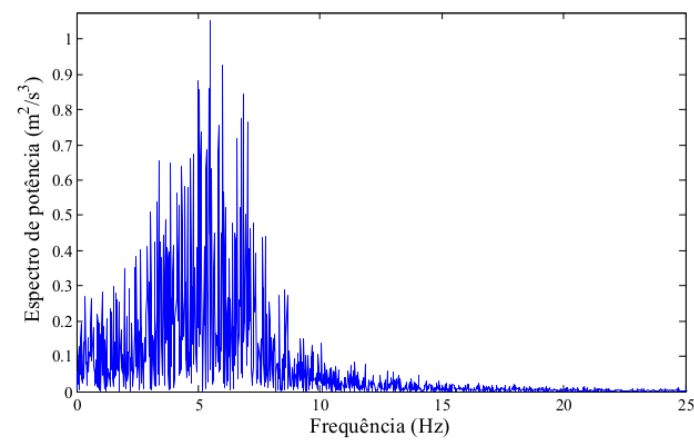

(c) Espectro de potência obtido na simulação 3.

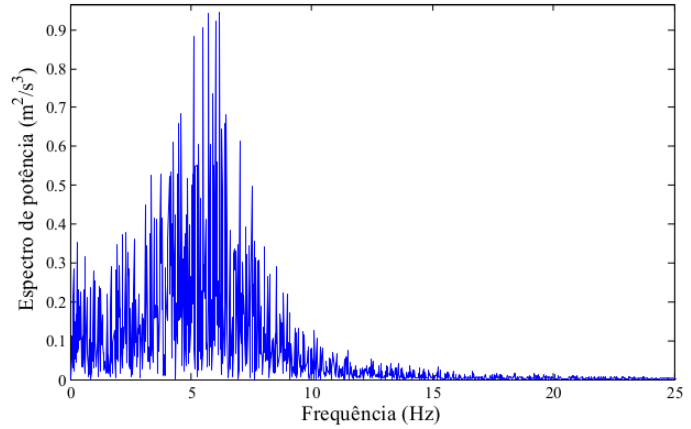

(b) Espectro de potência obtido na simulação 2 .

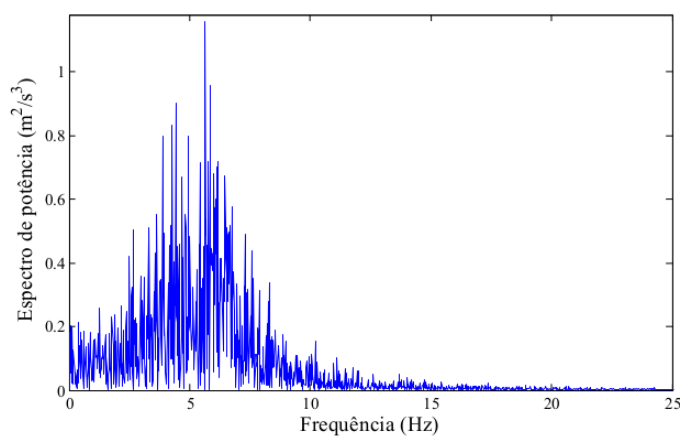

(d) Espectro de potência obtido na simulação 4 .

Pode-se verificar, que nas quatro simulações realizadas, os sinais sísmicos se assemelham aos obtidos por $[5]$.

Tendo os valores para a aceleração do solo, em cada instante considerado, calculou-se o vetor de forças externas, (9), e procedeu-se com a análise dinâmica estrutural que foi, então, realizada pelo método de Newmark, através da aplicação de (13), (15) e (16). Nessa etapa, considerou-se que a estrutura possuía 10 andares $(n=10)$ e que e o valor correspondente a massa, amortecimento e rigidez, em cada andar, são dados, respectivamente, por:

$$
m_{i}=360.000 \mathrm{Kg}, \quad k_{i}=650 \mathrm{MN} / \mathrm{m}, \quad c_{i}=6,2 \mathrm{MN} . \mathrm{s} / \mathrm{m}, \quad i=1,2, \ldots, n .
$$

As Tabelas 1, 2 e 3 mostram os resultados obtidos por [5] e pelos autores, referentes aos deslocamentos e acelerações máximas, em módulo, Tabelas 1 e 2, respectivamente, e os deslocamentos máximos, em módulo, entre os andares, ou seja, a diferença de deslocamentos entre $n$ e $n-1$, Tabela 3.

Conforme pode ser observado, através das Tabelas 1, 2 e 3, os maiores deslocamentos e acelererações ocorrem no décimo andar do edifício e o maior deslocamento entre andares é verificado entre o primeiro andar e a base da estrutura. As Figuras 7, 8 e 9 ilustram, nessa ordem, esses fatos, com referência à simulação 3. Conforme pode ser visualizado para um carregamento estocástico a resposta estrutural também será estocástica.

As Tabelas 1, 2 e 3, mostram que os resultados obtidos pelos autores são diferentes dos encontrados por [5], contudo, em termos de simulação de sinais sísmicos é importante ressaltar que tratando-se de um processo estocástico, tanto os valores encontrados por [5], como os valores obtidos neste trabalho representam um possível registro sísmico pertencente a um conjunto de registros sísmicos, cujas características de aleatoriedade são definidas conforme a seleção dos ângulos de fase, ou seja, cada vez que a rotina for executada diferentes soluções são encontradas. Também, é importante destacar que os valores máximos calculados de resposta estrutural, em cada simulação, referem-se apenas a uma execução da rotina e não a uma média de várias execuções. Sendo assim, apesar dos resultados serem diferentes eles apresentam diferenças aceitáveis, o que valida a rotina computacional implementada neste trabalho. 
Tabela 1: Deslocamento máximo por andar $(\mathrm{cm})$.

\begin{tabular}{|c|c|c|c|c|c|}
\hline Andar & Mohebbi et al. [5] & Simulação 1 & Simulação 2 & Simulação 3 & Simulação 4 \\
\hline 1 & 2,17 & 2,37 & 2,70 & 2,27 & 1,97 \\
\hline 2 & 4,30 & 4,53 & 5,22 & 4,43 & 3,87 \\
\hline 3 & 6,28 & 6,44 & 7,51 & 6,42 & 5,68 \\
\hline 4 & 8,01 & 8,11 & 9,56 & 8,19 & 7,36 \\
\hline 5 & 9,50 & 9,63 & 11,34 & 9,73 & 8,90 \\
\hline 6 & 10,75 & 11,19 & 12,80 & 11,23 & 10,24 \\
\hline 7 & 11,86 & 12,49 & 13,92 & 12,47 & 11,34 \\
\hline 8 & 12,88 & 13,47 & 14,78 & 13,41 & 12,19 \\
\hline 9 & 13,63 & 14,12 & 15,38 & 14,03 & 12,81 \\
\hline 10 & 14,03 & 14,45 & 15,69 & 14,35 & 13,14 \\
\hline
\end{tabular}

Tabela 2: Aceleração máxima por andar $\left(\mathrm{cm} / \mathrm{s}^{2}\right)$.

\begin{tabular}{|c|c|c|c|c|c|}
\hline Andar & Mohebbi et al. [5] & Simulação 1 & Simulação 2 & Simulação 3 & Simulação 4 \\
\hline 1 & 345,35 & 413,60 & 370,35 & 365,05 & 408,77 \\
\hline 2 & 571,60 & 629,80 & 626,97 & 595,59 & 640,10 \\
\hline 3 & 708,65 & 704,94 & 752,15 & 755,02 & 763,97 \\
\hline 4 & 708,89 & 662,30 & 817,04 & 827,92 & 821,15 \\
\hline 5 & 749,88 & 659,30 & 843,80 & 813,55 & 775,43 \\
\hline 6 & 719,11 & 658,42 & 913,45 & 724,94 & 704,56 \\
\hline 7 & 713,77 & 725,92 & 933,10 & 733,71 & 738,55 \\
\hline 8 & 713,73 & 800,44 & 916,71 & 798,35 & 739,02 \\
\hline 9 & 730,84 & 867,73 & 866,62 & 878,18 & 800,35 \\
\hline 10 & 802,68 & 908,58 & 905,12 & 922,76 & 857,62 \\
\hline
\end{tabular}

Tabela 3: Deslocamento máximo entre os andares $(\mathrm{cm})$.

\begin{tabular}{|c|c|c|c|c|c|}
\hline Andar & Mohebbi et al. [5] & Simulação 1 & Simulação 2 & Simulação 3 & Simulação 4 \\
\hline 1 & 2,17 & 2,37 & 2,70 & 2,27 & 1,97 \\
\hline 2 & 2,13 & 2,16 & 2,51 & 2,17 & 1,91 \\
\hline 3 & 1,99 & 1,95 & 2,31 & 2,01 & 1,82 \\
\hline 4 & 1,76 & 1,90 & 2,14 & 1,88 & 1,69 \\
\hline 5 & 1,64 & 1,78 & 1,88 & 1,72 & 1,53 \\
\hline 6 & 1,57 & 1,58 & 1,63 & 1,50 & 1,37 \\
\hline 7 & 1,38 & 1,30 & 1,38 & 1,29 & 1,23 \\
\hline 8 & 1,12 & 1,00 & 1,09 & 1,07 & 1,02 \\
\hline 9 & 0,79 & 0,70 & 0,75 & 0,77 & 0,73 \\
\hline 10 & 0,41 & 0,36 & 0,38 & 0,40 & 0,38 \\
\hline
\end{tabular}

\section{Considerações finais}

Este artigo foi elaborado com o intuito de apresentar os resultados de uma rotina computacional implementada no programa MATLAB ${ }^{\circledR}$, para a geração de uma excitação sísmica e análise da resposta dinâmica unidimensional de um edifício com $n$ GDL. Os resultados obtidos na seção anterior mostram que o objetivo foi alcançado com sucesso e, assim, a rotina computacional poderá ser utilizada como base para o desenvolvimento de trabalhos mais complexos, como por exemplo, estender o estudo considerando a excitação sísmica como um processo estocástico não-estacionário e aplicar esse tipo de excitação à estruturas tridimensionais, discretizadas pelo método dos elementos finitos. 
Figura 7: Deslocamento referente ao $10^{\circ}$ andar do edifício, relativo à simulação 3 .

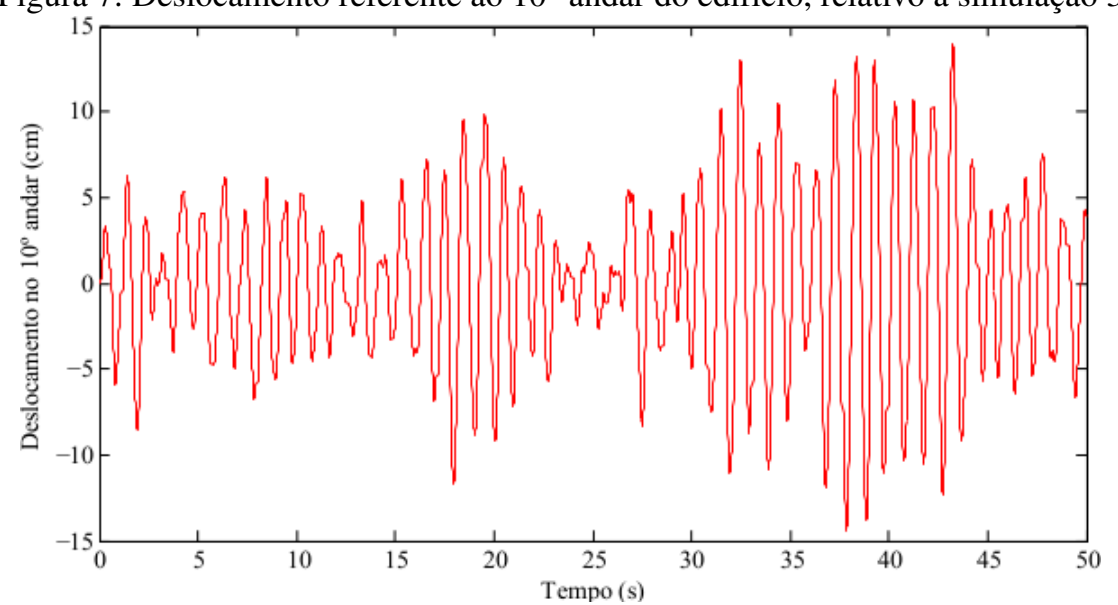

Figura 8: Aceleração referente ao $10^{\circ}$ andar do edifício, relativo à simulação 3 .

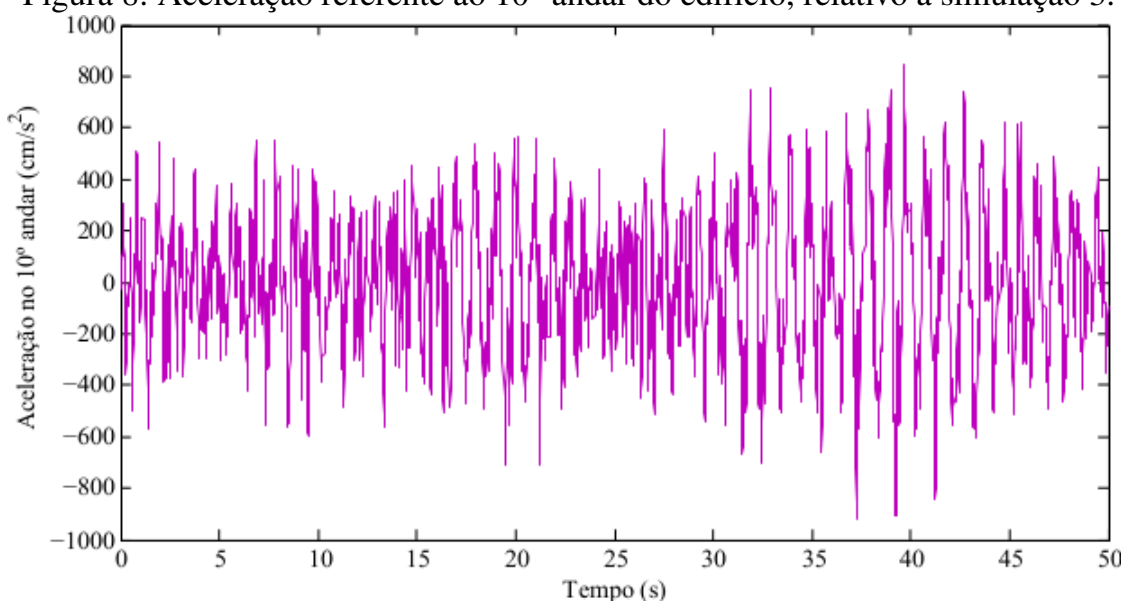

Figura 9: Deslocamento entre o $1^{\circ}$ andar e a base do edifício, relativo à simulação 3.

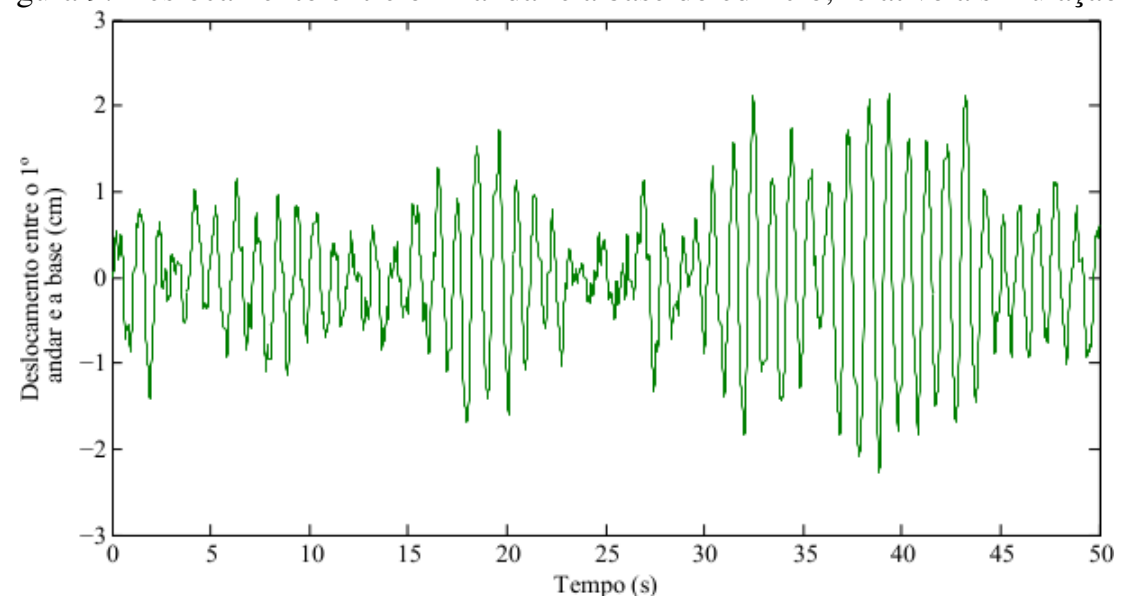




\section{Referências}

[1] USGS. United States Geological Survey. 2016. Disponível em: <http://earthquake.usgs.gov>. Acesso em: 17 jun. 2016.

[2] ABNT. NBR 15.421: Projeto de Estruturas Resistentes a Sismos-Procedimento. Rio de Janeiro, Brasil: [s.n.], 2006.

[3] ROBERTS, J. B.; SPANOS, P. D. Random Vibration and Statistical Linearization. Chichester, New York, Brisbane, Toronto, Singapore: Ed. John Wiley \& Sons, 1990.

[4] MIGUEL, L. F. F.; MIGUEL, L. F. F.; LOPEZ, R. H. Simultaneous optimization of force and placement of friction dampers under seismic loading. Engineering Optimization, v. 48, n. 4, p. 582-602, 2016. Disponível em: <http://dx.doi.org/10.1080/0305215X.2015.1025774>. Acesso em: 17 jun. 2016.

[5] MOHEBBI, M. et al. Designing optimal multiple tuned mass dampers using genetic algorithms (GAs) for mitigating the seismic response of structures. Journal de Vibration and Control, SAGE Journals, v. 19, n. 4, p. 605-625, 2013. ISSN 1741-2986. Disponível em: <http://journals.sagepub.com/doi/abs/10.1177/ $1077546311434520>$. Acesso em: 17 jun. 2016.

[6] SINGH, M. P.; MORESCHI, L. M. Optimal seismic response control with dampers. Earthquake Engineering and Structural Dynamics, v. 30, n. 4, p. 553-572, 2001. ISSN 1096-9845. Disponível em: <http://onlinelibrary.wiley.com/doi/10.1002/eqe.23/abstract>. Acesso em: 17 jun. 2016.

[7] KANAI, K. An empirical formula for the spectrum of strong earthquake motions. Bulletin Earthquake Research Institute University of Tokyo, n. 39, p. 85-95, 1961.

[8] TAJIMI, H. A statistical method of determining the maximum response of a building structure during an earthquake. In: Proceedings of 2nd World Conference in Earthquake Engineering (WCEE). Tóquio, Japão: [s.n.], 1960. v. 1, p. 781-797.

[9] SHINOZUKA, M.; JAN, C.-M. Digital simulation of random processes and its applications. Journal of Sound and Vibration, Elsevier Ltd., v. 25, n. 1, p. 111-128, 1972. ISSN 0022-460X. Disponível em: <http: //www.sciencedirect.com/science/article/pii/0022460X72906001>. Acesso em: 17 jun. 2016.

[10] AlbUQUERQUe, J. P. A.; FORTES, J. M. P.; FINAMORE, W. A. Probabilidade, Variáveis Aleatórias e Processos Estocásticos. Rio de Janeiro, Brasil: Ed. Interciência, 2008.

[11] BENDAT, J. S.; PIERSOL, A. G. Random Data - Analysis and Measurement Procedures. Chichester, New York, Brisbane, Toronto, Singapore: Ed. John Wiley \& Sons, 1986.

[12] NEWLAND, D. E. An Introduction to Random Vibrations and Spectral Analysis. New York1: John Wiley \& Sons, Inc., 1984.

[13] CLOUGH, R. W.; PENZIEN, J. Dynamics of Structures. New York: McGraw-Hill, Inc., 1993.

[14] RAO, S. S. Vibrações Mecânicas. S ao Paulo, Brasil: Pearson Prentice Hall, 2008. 